\title{
基于粗粒化模型对有机溶剂的分子动力学模拟
}

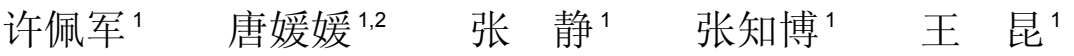 \\ 邵 颖 ${ }^{3}$ 沈虎峻 ${ }^{2, *}$ 毛英臣 ${ }^{1, *}$ \\ ('辽宁师范大学物理与电子技术学院, 辽宁大连 116029; 2 中国科学院大连化学物理研究所, 辽宁大连 116023; \\ ${ }^{3}$ 大连海事大学物理系, 辽宁 大连 116026)
}

\begin{abstract}
摘要: 在基于 Boltzmann 分布对四种基本构象进行 Monte Carlo 取样后, 通过与全原子模型的范德华势比较 得到了 Gay-Berne (GB)参数. 又在对用量化计算得到的分子体系的电势进行电荷、偶极矩和四极矩的拟合后, 得到了电多极展开势(EMP)参数. 利用得到的粗粒化参数, 基于粗粒化模型, 对 $\mathrm{CHCl}_{3}$ 及四氢呋喃(THF)两种有 机溶剂进行了分子动力学模拟(MDS), 并将结果同全原子模拟进行了比较. 计算结果表明用粗粒化模型从整体 上能重复全原子模型的模拟结果, 但在某些细节的计算与全原子模型有偏差, 其原因可能是目前工作仅考虑了 单位点情况, 为此今后在对具有复杂结构的分子进行粗粒化模拟时还应考虑合理放置及增加相互作用位点.
\end{abstract}

关键词：粗粒化模型； Gay-Berne势; 电多极展开势; 径向分布函数; 分子动力学模拟

中图分类号: $0645 ; 0641$

\section{Molecular Dynamics Simulation of Organic Solvents Based on the Coarse-Grained Model}

\author{
XU Pei-Jun ${ }^{1} \quad$ TANG Yuan-Yuan ${ }^{1,2} \quad$ ZHANG Jing ${ }^{1} \quad$ ZHANG Zhi-Bo ${ }^{1} \quad$ WANG Kun ${ }^{1}$ \\ SHAO Ying ${ }^{3}$ SHEN Hu-Jun ${ }^{2, *}$ MAO Ying-Chen ${ }^{1, *}$ \\ ('School of Physics and Electronic Technology, Liaoning Normal University, Dalian 116029, Liaoning Province, P. R. China; \\ ${ }^{2}$ Dalian Institute of Chemical Physics, Chinese Academy of Sciences, Dalian 116023, Liaoning Province, P. R. China; \\ ${ }^{3}$ Department of Physics, Dalian Maritime University, Dalian 116026, Liaoning Province, P. R. China)
}

\begin{abstract}
To obtain Gay-Berne (GB) parameters, we carried out Monte Carlo sampling of four reference configurations based on the Boltzmann distribution. After comparing with the van der Waals potential within the all-atom model we obtained the GB parameters. Also by fitting the charge, dipole, and quadrupole with the electric potential obtained from quantum chemical computations with Gaussian 03 we obtained the electric multipole potential (EMP) parameters. With the GB-EMP parameters we then carried out molecular dynamics simulations (MDS) for $\mathrm{CHCl}_{3}$ and tetrahydrofuran (THF) based on the coarsegrained (CG) model. Compared with the all-atom model, the CG model can reproduce the simulation results on the whole, but there are some deviations in the simulations in some details. The reason is that we only take one interaction site into account in this work. Therefore, for more complicated molecules it is necessary to take the placement of the interaction sites into account. Additionally, the multi-sites situation is also considered in the MDS within the frame of the coarse-grained model.
\end{abstract}

Key Words: Coarse-grained model; Gay-Berne potential; Electric multipole potential; Radial distribution function; Molecular dynamics simulation

Received: February 28, 2011; Revised: May 9, 2011; Published on Web: June 14, 2011.

"Corresponding authors. MAO Ying-Chen, Email: myc@Innu.edu.cn; Tel: +86-411-82158367. SHEN Hu-Jun, Email: hshen@dicp.ac.cn; Tel: +86-411-84379875.

The project was supported by the Fundamental Research Funds for the Central Universities, China (2009QN069).

中央高校专项资金优秀青年教师基金(2009QN069)资助项目

(C) Editorial office of Acta Physico-Chimica Sinica 


\section{1 引言}

分子动力学模拟(MDS)已经成为了研究复杂分 子体系的重要工具, 尽管一些计算方法和技术手段 已经取得了突破, ${ }^{1-3}$ 但当前基于全原子模型对某些 较大体系的动力学模拟时间尺度仍然非常短, 并不 能满足与实验比较的迫切需要..$^{4-6}$ 已知在利用经验 力场进行的全原子分子动力学模拟主要包括两部 分:一是对分子的构象进行统计分析; 二是结合相 应的构象分析体系的能量及受力情况..$^{7-9}$ 而对某一 分子体系, 由于非键结的范德华相互作用项和静电 相互作用项的数目远远大于键长、键角和二面角相 互作用项的数目, 因此对范德华相互作用和静电相 互作用的计算将要耗费大量的计算时间. 为了简化 对这两种相互作用的计算, 研究人员已提出了多种 粗粒化模型 $\left(C G\right.$ 模型). ${ }^{10-14} \mathrm{CG}$ 模型的主要特点在于 它将研究体系中的分子或分子的某一部分近似看 成一个原子团簇, 该原子团簇内部信息(键长、键角、 二面角等)被屏蔽, 即该团簇被处理为没有内部结构 的“粒子”, 该粒子对应于全原子模型中的单个原 子. 而在利用 $\mathrm{CG}$ 模型进行分子动力学模拟之前还 必须获得描述这些粒子间范德华相互作用和静电 相互作用的力场参数, 应当指出这一部分工作也是 构建 $C G$ 模型的关键部分. CG 模型结合了日益发展 的计算机水平, 使得对更大更复杂体系的长时模拟 成为可能.

该方法的主要缺陷就在于它的计算精度要低 于基于全原子模型的分子动力学模拟..$^{10}$ 此外还需 指出的是大多数的 $\mathrm{CG}$ 模型仅对某些特殊体系适 用, 而不能应用于其他体系, 即模型的可移植性有 待提高. 产生这一问题的主要原因在于多数 CG 模 型将粗粒化粒子看成是球形, 这样导致了这些模型 没能更好地描述分子间的范德华相互作用, 以及未 能对由此而导致的电势空间分布改变的静电相互 作用作出更好的描述. 为了克服这些缺陷, 改进 $\mathrm{CG}$ 模型, Golubkov 和 Ren ${ }^{14}$ 提出了在计算粗粒化粒子间 相互作用时, 必须考虑由粗粒化粒子的形状而引起 的 Gay-Berne (GB) 效应及电多极矩效应, 这些改进 在他们对水、苯及甲醇三种构象相对简单的分子体 系的动力学模拟中得到了较理想体现. 此外也应认 识到的是他们工作的基石就在于较好地拟合得到 了描述上述三种分子的粗粒化力场参数. 为了检验 如何利用 $\mathrm{CG}$ 模型对复杂分子体系进行高效计算, 本文采用类似方法对 $\mathrm{CHCl}_{3}$ 及四氢呋喃(THF) 分子
的两种构象在相对复杂的有机溶剂中进行了分子 动力学模拟.

\section{2 计算与模拟方法}

\section{1 描述非球形分子的 Gay-Berne 势和电多极展 开势(EMP)}

任何一个好的模型必须具备两个主要特征,一 方面数学上要简单并易于处理, 另一方面能够对模 拟体系做较好的物理描述. 基于这两方面的考虑, Berne 和 Pechukas ${ }^{15}$ 近似地将分子视为绕其主轴旋转 的椭球体, 得到了由高斯函数描述的经验势. 在对非 球形分子的分子模拟中, 短距离吸引和排斥相互作 用可通过如下方法来完成, 即在分子内定义多个位 点, 每两个位点间的范德华相互作用势由 LennardJones 势描述. 需指出的是对于较大分子, 计算势的 时间会随位点数目的平方而递增, 从而使得计算效 率大为降低. 为了解决这一问题, Gay 和 Berne ${ }^{16}$ 对描 述非球形分子间的短距离排斥和相互吸引作用的 高斯重叠势进行了修正, 把分子处理成软的、单轴 的椭球体, 把 GB位点放置在椭球体的质心上, 用单 位点势替代多位点势, 这样就得到了 GB势. 在惯性 系中, 椭球体在位形空间中由其质心坐标和三个欧 拉角描述, 对应于由一套 GB参数来描述. 目前, GB 势在描述液晶体系中得到了广泛应用. ${ }^{.7-20}$ 为了更好 地控制 GB势的软硬度, Brene和 Pechukas 建议增加 参数来控制经验势的柔性, 由此 Kabadi 等 ${ }^{21,22}$ 引入了 $d_{\mathrm{w}}$ 参数.

两个粗粒化粒子间的 GB 势可表示为

$$
\begin{aligned}
U_{\mathrm{GB}}\left(\hat{u}_{i}, \hat{u}_{j}, \hat{r}_{i j}\right)=4 \varepsilon\left(\hat{u}_{i}, \hat{u}_{j}, \hat{r}_{i j}\right) \times & \\
& {\left[\left(\frac{d_{\mathrm{w}} \sigma_{0}}{r_{i j}-\sigma\left(\hat{u}_{i}, \hat{u}_{j}, \hat{r}_{i j}\right)+d_{\mathrm{w}} \sigma_{0}}\right)^{12}-\right.} \\
& \left.\left(\frac{d_{\mathrm{w}} \sigma_{0}}{r_{i j}-\sigma\left(\hat{u}_{i}, \hat{u}_{j}, \hat{r}_{i j}\right)+d_{\mathrm{w}} \sigma_{0}}\right)^{6}\right]
\end{aligned}
$$

上式中的距离参数 $\sigma\left(\hat{u}_{i}, \hat{u}_{j}, \hat{r}_{i j}\right)$ 可取如下形式

$$
\begin{aligned}
\sigma\left(\hat{u}_{i}, \hat{u}_{j}, \hat{r}_{i j}\right)= & \sigma_{0}\left[1-\left\{\frac{\chi \alpha^{2}\left(\hat{u}_{i} \cdot \hat{r}_{i j}\right)^{2}+\chi \alpha^{-2}\left(\hat{u}_{j} \cdot \hat{r}_{i j}\right)^{2}}{1-\chi^{2}\left(\hat{u}_{i} \cdot \hat{u}_{j}\right)^{2}}-\right.\right. \\
& \left.\left.\frac{2 \chi^{2}\left(\hat{u}_{i} \cdot \hat{r}_{i j}\right)\left(\hat{u}_{j} \cdot \hat{r}_{i j}\right)\left(\hat{u}_{i} \cdot \hat{u}_{j}\right)}{1-\chi^{2}\left(\hat{u}_{i} \cdot \hat{u}_{j}\right)^{2}}\right\}\right]^{-1 / 2}
\end{aligned}
$$


其中

$$
\begin{aligned}
& \sigma_{0}=\sqrt{d_{i}^{2}+d_{j}^{2}} \\
& \chi \alpha^{2}=\frac{l_{i}^{2}-d_{i}^{2}}{l_{i}^{2}+d_{j}^{2}} \\
& \chi \alpha^{-2}=\frac{l_{j}^{2}-d_{j}^{2}}{l_{j}^{2}+d_{i}^{2}} \\
& \chi^{2}=\frac{\left(l_{i}^{2}-d_{i}^{2}\right)\left(l_{j}^{2}-d_{j}^{2}\right)}{\left(l_{j}^{2}+d_{i}^{2}\right)\left(l_{i}^{2}+d_{j}^{2}\right)}
\end{aligned}
$$

其中 $l$ 和 $d$ 分别描述分子的长和宽, 这样分子的形状 就可以用任意的棒状、盘状或者球状来表示.

(1) 式中的阱深参数取如下形式

$$
\varepsilon\left(\hat{u}_{i}, \hat{u}_{j}, \hat{r}_{i j}\right)=\varepsilon_{0} \varepsilon_{1}{ }^{v}\left(\hat{u}_{i}, \hat{u}_{j}\right) \varepsilon_{2}{ }^{\mu}\left(\hat{u}_{i}, \hat{u}_{j}, \hat{r}_{i j}\right)
$$

其中 $\mu$ 和 $v$ 均为可调指数, 借鉴 Golubkov 和 $\operatorname{Ren}^{14}$ 的 工作, 本文分别将其取值为 2.0 和 1.0. 而 $\varepsilon_{1}$ 和 $\varepsilon_{2}$ 可分 别表示为

$$
\begin{aligned}
\varepsilon_{1}\left(\hat{u}_{i}, \hat{u}_{j}\right)=\left[1-\chi^{2}\left(\hat{u}_{i} \cdot \hat{u}_{j}\right)^{2}\right]^{-1 / 2} & \\
\varepsilon_{2}\left(\hat{u}_{i}, \hat{u}_{j}, \hat{r}_{i j}\right)= & 1-\left\{\frac{\chi^{\prime} \alpha^{\prime 2}\left(\hat{u}_{i} \cdot \hat{r}_{i j}\right)^{2}+\chi^{\prime} \alpha^{\prime-2}\left(\hat{u}_{j} \cdot \hat{r}_{i j}\right)^{2}}{1-\chi^{\prime 2}\left(\hat{u}_{i} \cdot \hat{r}_{i j}\right)^{2}}-\right. \\
& \left.\frac{2 \chi^{\prime 2}\left(\hat{u}_{i} \cdot \hat{r}_{i j}\right)\left(\hat{u}_{j} \cdot \hat{r}_{i j}\right)\left(\hat{u}_{i} \cdot \hat{u}_{j}\right)}{1-\chi^{2}\left(\hat{u}_{i} \cdot \hat{u}_{j}\right)^{2}}\right\}
\end{aligned}
$$

(9)式中的 $\chi^{\prime}$ 和 $\alpha^{\prime}$ 分别取如下形式

$$
\begin{aligned}
\chi^{\prime} & =\frac{1-\left(\varepsilon_{\mathrm{E}} / \varepsilon_{\mathrm{S}}\right)^{1 / \mu}}{1+\left(\varepsilon_{\mathrm{E}} / \varepsilon_{\mathrm{S}}\right)^{1 / \mu}} \\
\alpha^{\prime 2} & =\left[1+\left(\varepsilon_{\mathrm{E}} / \varepsilon_{\mathrm{S}}\right)^{1 / \mu}\right]^{-1}
\end{aligned}
$$

其中, $\varepsilon_{0}$ 描述了交叉结构的阱深, $\varepsilon_{\mathrm{E}}$ 和 $\varepsilon_{\mathrm{S}}$ 分别表示 尾对尾和边对边结构的阱深. 当两个分子相同或 者其中一个分子是球形分子时, 势函数用 Berne 和 Pechukas 的原始形式表示. 如果两个分子都是球形 的, 势函数将进一步简化为 Lennard-Jones 势.

长距离静电相互作用可以由电多极展开势来 表示. 在大于分子尺寸的距离处, 分子的电势可准 确地通过对其质心的电多极展开势来表示 ${ }^{23}$

$$
M=\left(q, \mu_{x}, \mu_{y}, \mu_{z}, \Theta_{x x}, \Theta_{x y}, \ldots, \Theta_{z z}\right)
$$

其中 $q 、 \mu 、 \Theta$ 分别表示电荷、偶极矩和四极矩. 电多极 势可表示为电荷 - 电荷相互作用势、电荷-偶极相互 作用势、电荷-四极相互作用势等项之和. 本文在分 子的质心上放置了一个电多极展开位点, 同时选取
了和 GB 位点相同的惯性系.

两个多极位点间的相互作用可通过电多极展 开势表示为

$$
U_{\mathrm{EMP}}=M_{i}^{t} T_{i j} M_{j}
$$

其矩阵形式可参见文献. ${ }^{14,24}$ 本文对静电相互作用截 断值的处理方法, 采用了 TINKER 软件的标准方 法. ${ }^{25-27}$

此外还需指出的是, 考虑到由于粗粒化粒子具 有各向异性的形状而使得在进入排斥的范德华相 互作用区域前电多极展开方法将给出不正确的相 互作用势, 类似于文献, ${ }^{14,28}$ 本文同样加入了一个阻 尼函数以确保对短距离多极相互作用势的精确描 述.

\section{2 粗粒化模型力场参数的确定}

\subsubsection{Gay-Berne (GB) 参数的拟合}

本文利用粗粒化模型进行分子模拟的前提是 要获得 GB-EMP 模型的参数, 为此我们首先对研究 体系进行了合理取样选取, 然后利用描述有机分子 的 AMBER 力场 (GAFF) ${ }^{29,30}$ 通过与全原子的范德华 势进行比较后拟合得到了 GB 参数. 选取 $\mathrm{GAFF}$ 力场 是由于它的函数形式简单, 原子类型有限, 还可利 用实验和理论模型计算获得力常数和部分原子电 荷. 在研究中, 我们发现构象的选取会直接影响 GB 参数的拟合, 进而决定 GB 势是否能准确地模拟全

表 1 氯仿和 THF 分子的四种基本构象

Table 1 Four reference configurations of $\mathrm{CHCl}_{3}$ and THF molecules

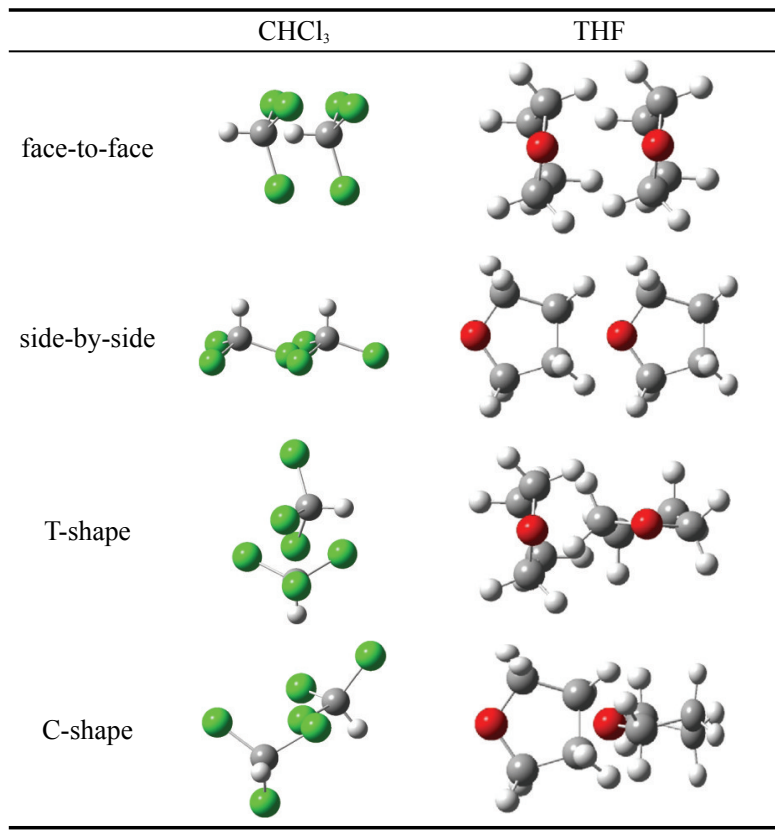


原子的范德华势, 因此选择可以反映真实分子液体 环境的构象是十分重要的. 由于在实际的溶液环境 中, 面对面(或者尾对尾)、边对边、 $\mathrm{T}$ 形和交叉结构这 四种构象出现的几率相对较大, ${ }^{17,19}$ 另外考虑到在真 实的液体环境中分子的构象趋于取能量最低的构 象, 所以本文在篎选用于拟合 $\mathrm{GB}$ 参数的构象时, 对 上述四种构象分别基于 Boltzmann 分布采用 Monte Carlo方法获得到分子的构象集. 这样才可确保在一 定的能量范围内, 构象数目随能量的升高而减少. 表 1 给出了 $\mathrm{CHCl}_{3}$ 和 $\mathrm{THF}$ 这两种分子的四种基本构 象.

图 1 及图 2 给出了氯仿和 $\mathrm{THF}$ 分子的四种基本 构象在各能量区间内的构象数目. 从图中我们可以 清楚地看到所选的分子构象在较低的能量范围内
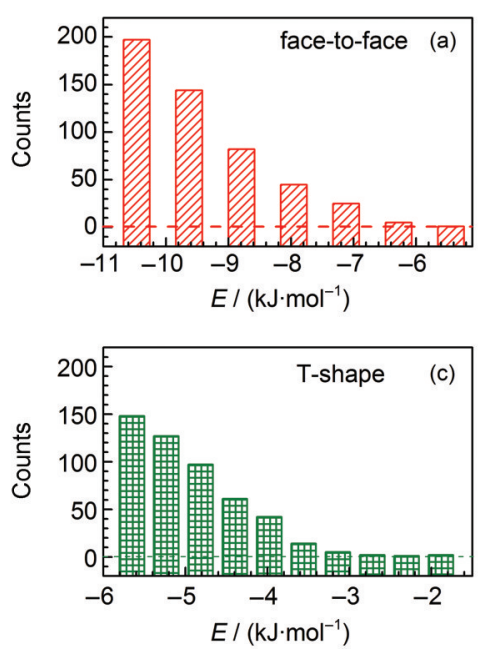

表 2 氯仿和 THF 分子的 GB 参数

Table 2 GB parameters of $\mathrm{CHCl}_{3}$ and THF molecules

\begin{tabular}{cccccc}
\hline & $l / \mathrm{nm}$ & $d / \mathrm{nm}$ & $\varepsilon_{0} /\left(\mathrm{kJ} \cdot \mathrm{mol}^{-1}\right)$ & $\varepsilon_{\mathrm{E}} / \varepsilon_{\mathrm{s}}$ & $d_{\mathrm{w}}$ \\
\hline $\mathrm{CHCl}_{3}$ & 0.247 & 0.402 & 3.182 & 2.29 & 0.66 \\
$\mathrm{THF}$ & 0.313 & 0.403 & 3.977 & 1.37 & 0.61 \\
\hline
\end{tabular}

较多, 且构象数随着能量的升高而逐渐减小.

在参数拟合的具体过程中, 5 个自由参数 $(l, d$, $\left.\varepsilon_{0}, \varepsilon_{\mathrm{E}} / \varepsilon_{\mathrm{s}}, d_{\mathrm{w}}\right)$ 由遗传算法拟合得到. 表 2 给出用上述方 案拟合得到的氯仿和 THF 分子的 GB 参数.

\subsubsection{EMP 的参数拟合}

在拟合粗粒化模型的 EMP 参数时, 本文首先应 用 Gaussian 03 软件包 ${ }^{31}$ 的 MP2 方法采用 6-311G** $(2 d, 2 p)$ 机组计算得到了两种分子的电势, 然后利用 GDMA 程序 ${ }^{32}$ 对电势进行电荷、偶极和四极的拟合,
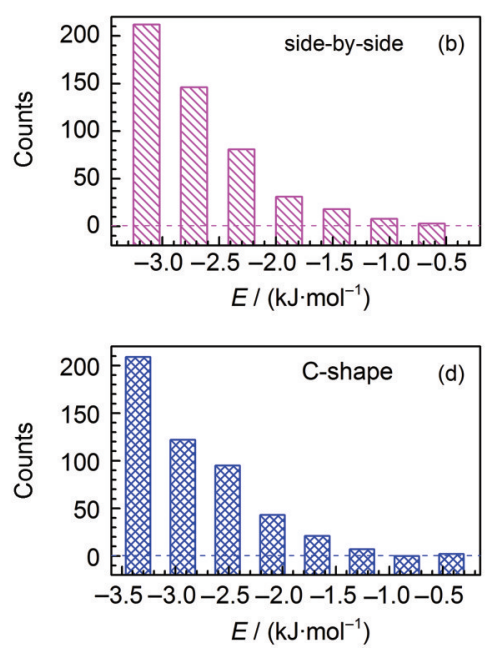

图 1 氯仿分子的构象数目随能量的变化

Fig.1 Configuration numbers of $\mathrm{CHCl}_{3}$ molecular change with the energy The dash lines are only guides to the eyes.
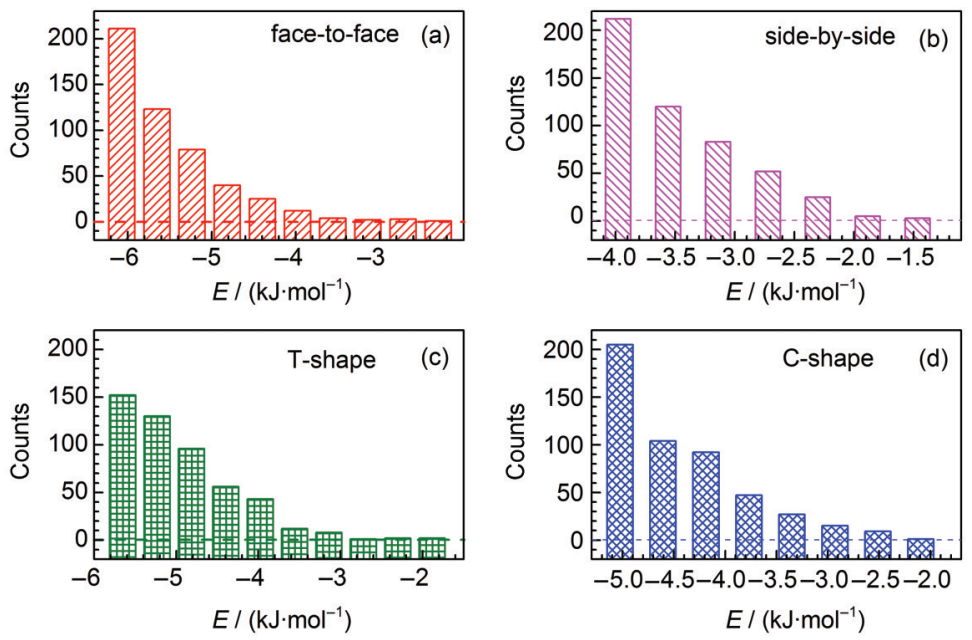

图 2 THF 分子的构象数目随能量的变化

Fig.2 Configuration numbers of THF molecular change with the energy The dash lines are only guides to the eyes. 
表 3 氯仿和 THF 分子的 EMP 参数

Table 3 EMP parameters of $\mathrm{CHCl}_{3}$ and THF molecules

\begin{tabular}{|c|c|c|c|c|c|c|}
\hline & Charge/C & $10^{21}$ Dipole $/(\mathrm{C} \cdot \mathrm{nm})$ & \multicolumn{3}{|c|}{$10^{22}$ Quadrupole $/\left(\mathrm{C} \cdot \mathrm{nm}^{2}\right)$} & Inertia $/\left(\mathrm{g} \cdot \mathrm{nm}^{2} \cdot \mathrm{mol}^{-1}\right)$ \\
\hline \multirow[t]{3}{*}{$\mathrm{CHCl}_{3}$} & 0 & 2.523 & -0.517 & -0.144 & -1.718 & 15560.9 \\
\hline & 0 & 0.516 & -0.144 & 0.155 & -0.352 & 15561.5 \\
\hline & 0 & 1.373 & -1.718 & -0.352 & 0.362 & 30030.0 \\
\hline \multirow[t]{3}{*}{ THF } & 0 & 1.804 & -4.660 & -0.003 & 0.000 & 7031.8 \\
\hline & 0 & 0.001 & -0.003 & 3.515 & 0.163 & 7116.7 \\
\hline & 0 & 0.000 & 0.000 & 0.163 & 1.145 & 12475.5 \\
\hline
\end{tabular}

最后得到了两种分子粗粒化模型的 EMP 参数. 表 3 中给出了氯仿和 THF 的 EMP 参数.

\section{3 模拟体系与动力学模拟方法}

为了检验本文对氯仿和 THF 分子粗粒化模型 GB-EMP 参数的拟合, 我们利用粗粒化模型对两种 有机分子溶剂进行了粗粒化的分子动力学模拟. 为 了在计算的过程中可任意地在粗粒化模型和全原 子模型间进行转换, 方便容易地比较 CG 模型和全 原子模型的结果, 故本文在计算中采用了 TINKER 软件包. 此外还基于如下考虑, 即 TINKER也包含了 对粗粒化粒子 GB-EMP 能量、力和力矩部分的计 算, 并可在惯性系中非常容易地整合 GB 和 EMP两 部分的计算结果.

氯仿和 THF 有机溶剂的立方体盒子由 Materials Studio (MS) 5.0 软件生成, 其边长均为 $3 \mathrm{~nm}$. 对 $\mathrm{GB}$ 和 $\mathrm{EMP}$ 两部分的截断半径都取为 $1.2 \mathrm{~nm}$ (这考 虑到在通常情况下, 要求截断半径值不大于盒子边 长的一半). $\mathrm{CG}$ 模型和全原子模型的分子动力学模 拟都是采用 NVT 系综在室温 $(298 \mathrm{~K})$ 下进行的. 基于 全原子模型的分子动力学模拟的计算步长取为 1 fs. 文献 ${ }^{14}$ 对考虑了 GB-EMP 效应的 CG 模型的时间 步长作了实验, 推荐 $\mathrm{CG}$ 模型的计算步长可取 5-20 fs. 考虑到由于自由度的减小, 以及分子内高频运 动的“屏蔽”, 在本文我们取时间步长为 $5 \mathrm{fs}$. 在对 运动方程进行数值化处理时, 两种模型的计算都采 用了 TINKER 推荐的 BERMAN 积分算法. ${ }^{25}$

\section{3 结果与讨论}

\section{1 对范德华相互作用的分析}

在图 3 中, 我们比较了分别利用 $C G$ 模型和全原 子模型计算得到的氯仿分子的范德华相互作用势, 可以看到 CG 模型结果与全原子模型结果总体符合 得较好. 对面对面及交叉结构, 两种方法得到的作 用曲线几乎完全相同; 而对于边对边及 $\mathrm{T}$ 型构象, 可 以看出相比于全原子模型, 粗粒化模型得到了较小
的阱深和距离参数, 这一现象对 $\mathrm{T}$ 型构象尤为明显. 此外, 基于粗粒化模型对四种构象的计算结果整体 趋势较好地符合了 Gay 及 Berne ${ }^{16}$ 的结论.

通过将分子构象的取样图(图 1)与范德华相互 作用势能曲线图(图 3)进行比较, 可以发现分子构象 数最多的能量区域正好对应于范德华相互作用势 能曲线图的最低点区域, 这也印证了分子构象都趋 于位于能量最低的构象状态的结论. ${ }^{19}$

图 4给出了基于两种模型对 THF 分子范德华相 互作用的比较. 从图中可以看出, 相比于结构简单 的氯仿分子, 对 THF 分子进行粗粒化模型的计算结 果与全原子结果有较大的偏差. 这表现在对应于四 种基本构象的阱深参数和距离参数都一致性地小 于全原子模型的计算结果. 从图 3 和图 4 中, 我们也 观察到两种分子的交叉结构和边对边结构的范德 华作用曲线非常接近, 几乎重合, 这一现象很好地 支持了文献 ${ }^{16}$ 的结论. 在对粗粒化参数做多次拟合 后, 计算结果仍然如此, 由此我们认为出现这一现 象的主要原因是我们仅考虑了单位点情况. 这也建

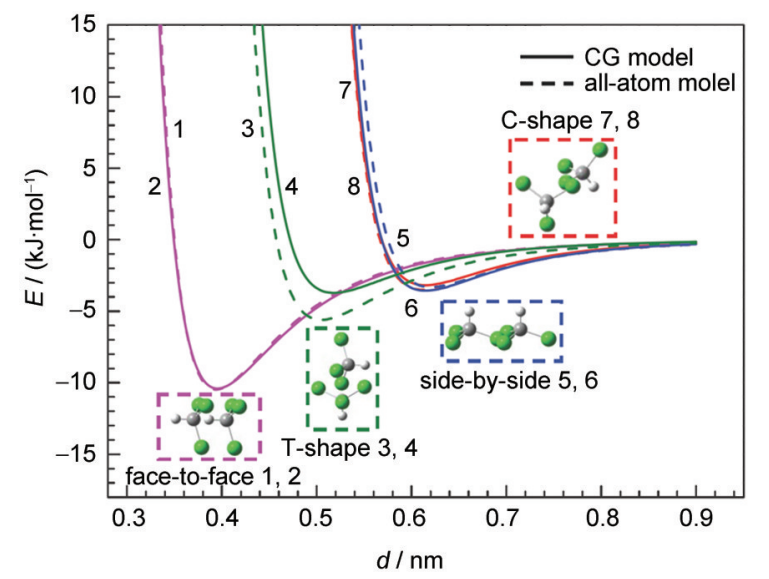

图 3 基于粗粒化模型和全原子模型对氯仿分子的范德华 相互作用曲线的比较

Fig.3 Comparison of van der Waals potential of $\mathrm{CHCl}_{3}$ based on the CG model and the all-atom model

The curves of four configurations, namely face to face (lines 1 and 2), side by side (lines 5 and 6), T-shape (lines 3 and 4), and cross shape (lines 7 and 8), are also displayed, respectively. 


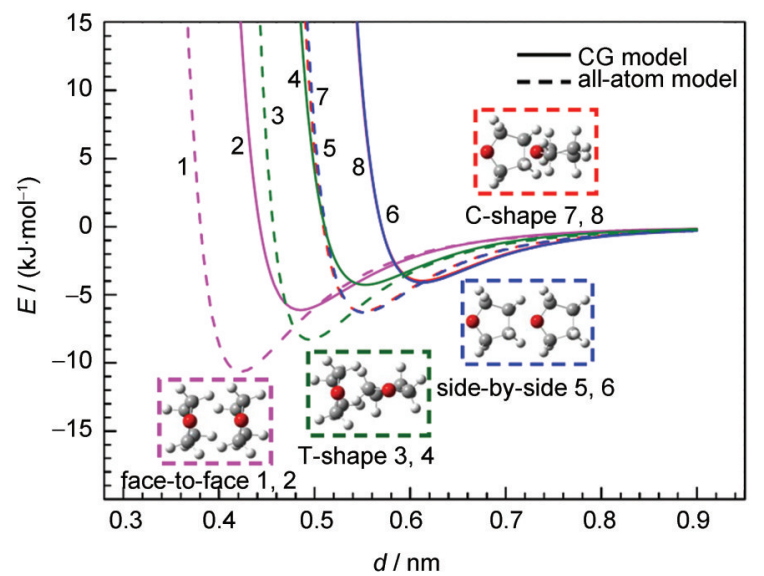

图 4 基于粗粒化模型和全原子模型对 THF 分子的范德 华相互作用曲线的比较

Fig.4 Comparison of van der Waals potential of THF based on the CG model and the all-atom model

The curves of four configurations, namely face to face (lines 1 and 2), side by side (lines 5 and 6), T-shape (lines 3 and 4) and cross shape (lines 7 and 8), are also displayed, respectively. The results of the CG model are represented with solid lines, and the dash lines are the results of the all-atom model.

议在用粗粒化模型对复杂结构的分子来计算分子 间范德华相互作用时应考虑增加相互作用位点.

考虑到粗粒化模型的普遍特征, ${ }^{10}$ 在目前阶段 的工作中, 要进一步检验粗粒化模型的计算效率, 还需结合其他物理量, 如溶剂的径向分布曲线等来 做进一步研究.

\section{2 径向分布函数的比较分析}

需要指出, 为了比较两种模型得到的径向分布 函数曲线, 首先需要把用粗粒化模型得到的 $\mathrm{CG}$ 轨 迹转换为全原子轨迹. 图 5 给出了氯仿分子的径向 分布函数曲线. 从图中可以看出, 尽管时间步长增 大为 $5 \mathrm{fs}$, 粗粒化的分子动力学模拟仍能给出一条 稳定的轨迹, 这说明 CG 模型能够较好地重复全原 子的模拟结果. 与全原子模型相比, $\mathrm{CG}$ 模型的径向
分布函数曲线起伏较少, 这符合粗粒化模型的基本 特征. 从 $\mathrm{C}-\mathrm{H}$ 径向分布曲线可看出, 在 $0.65 \mathrm{~nm}$ 处 $\mathrm{CG}$ 模型得到一波峰, 此外从 $\mathrm{Cl}-\mathrm{Cl}$ 及 $\mathrm{H}-\mathrm{Cl}$ 径向 分布曲线还可看出 $\mathrm{CG}$ 模型不能重现 $0.30 \mathrm{~nm}$ 处的 波峰, 但当距离较大时, $\mathrm{CG}$ 模型和全原子模型的结 果符合得较好. 在距离较小处, 两者的结果符合得 较差, 其原因可能与 EMP 相互作用位点的放置有 关. 在本文中, 与 GB 相互作用位点的放置相同, EMP 作用位点放置在粗粒化粒子的质心上, 这样就 造成了对电多极展开势空间分布描述的偏差, 从而 影响了对电负性较强元素 ( $\mathrm{Cl}$ 元素)的相关径向分 布函数曲线的模拟. 而我们注意到 Golubkov 和 Ren 等 ${ }^{33}$ 在最近实验工作中把 EMP 作用位点放到了其 他位置上, 相比于他们之前工作, ${ }^{14}$ 这样更好地模拟 了所研究体系的相关性质. 这也提示在以后的工作 中, 应针对分子的具体结构, 考虑把 EMP 位点放在 其他位置(如 $\mathrm{C}$ 原子上)而不是分子的质心, 或者考 虑增加额外的相互作用位点.

图 6 给出了 $\mathrm{THF}$ 溶剂的径向分布函数曲线. 可 以看出 $\mathrm{CG}$ 模型能较好地重复全原子的模拟结果, 然而必须指出的是, 与两种方法得到的范德华相互 作用曲线的较大差异不同, 两种模型的模拟结果符 合得较好, 没有出现类似于氯仿的径向分布曲线中 的较大差异. 鉴于分子结构的差异, 相比于将 EMP 相互位点放置在氯仿粗粒化粒子的质心上, 将该位 点放置在 THF 粗粒化粒子的质心上, 目前我们认为 是一种可以接受的处理方式. 对于 $\mathrm{CG}$ 模型仍然不 能较好地重复全原子模型在 $0.30 \mathrm{~nm}$ 处的较小起 伏, 我们认为考虑增加相互作用位点或者适当地调 整EMP 作用位点的位置会提高粗粒化模型的计算 精度.

通过 CG 模型进行分子动力学模拟的主要优点

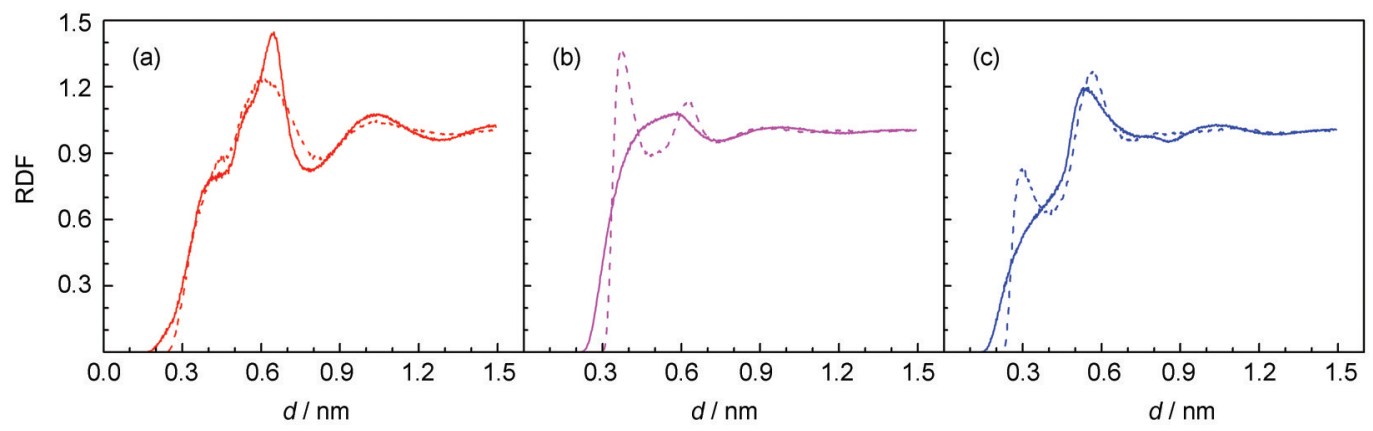

图 5 对基于两种模型得到的氯仿溶剂的径向分布函数(RDF)曲线的比较

Fig.5 Comparison of radial distribution functions (RDF) of $\mathrm{CHCl}_{3}$ solvent based on the two models (a) $\mathrm{C}-\mathrm{H}$, (b) $\mathrm{Cl}-\mathrm{Cl}$, (c) $\mathrm{H}-\mathrm{Cl}$; The results of the $\mathrm{CG}$ model are represented with solid lines, and the dash lines are the results of the all-atom model. 


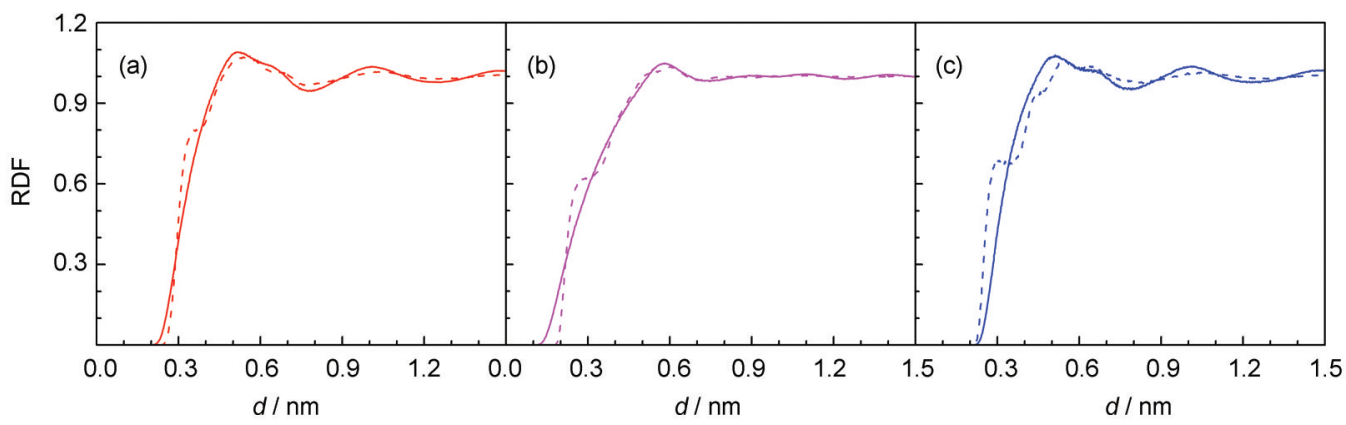

图 6 对基于两种模型得到的 THF 溶剂的径向分布函数(RDF)曲线的比较

Fig.6 Comparison of radial distribution functions (RDF) of THF solvent based on the two models

(a) $\mathrm{C}-\mathrm{H}$, (b) $\mathrm{H}-\mathrm{H}$, (c) $\mathrm{O}-\mathrm{H}$; The results of the $\mathrm{CG}$ model are represented with solid lines, and the dash lines are the results of the all-atom model.

是可以在保证一定精度的条件下较大幅度地提高 计算的效率, 这主要是由于用粗粒化的 GB-EMP 相 互作用代替了多对原子间的相互作用, 使得自由度 数得到了较大地减少. 此外一重要因素便是由于忽 略了键长、键角和二面角的高频运动, 而使得模拟 的时间步长增大了.

还需指出的是, 由于在处理 Gay-Berne 效应中 引入的 $d_{\mathrm{w}}$ 参数较好地控制了 $\mathrm{GB}$ 势的柔性, 所以用 本文中的 CG 模型还可用来模拟更大的复杂分子体 系. 此外在考虑了能更精确地描述静电相互作用的 电多极势效应后, 使得基于 CG 模型模拟那些带高 电量的生物大分子体系成为可能.

\section{4 结 论}

为了精确描述非球形分子间的相互作用, 考虑 了 Gay-Berne 效应和电多极势效应, 基于粗粒化模 型对氯仿和 THF 两种有机溶剂进行了分子动力学 模拟, 并同全原子模拟结果进行了比较. 为此本文 首先对研究体系的分子构象进行了合理取样, 然后 利用描述有机分子的 GAFF 力场, 通过与全原子的 范德华势进行比较后, 用遗传算法拟合得到了 GB 参数. 在拟合 $\mathrm{GB}$ 参数的过程中, 我们也注意到构象 选取对计算结果的影响, 针对 $\mathrm{CHCl}_{3}$ 和 THF 两种有 机溶剂中面对面、边对边、 $\mathrm{T}$ 型及交叉结构这四种构 象出现几率相对较大, 及分子构象都趋于位于能量 最低的构象状态的特征, 利用 Monte Carlo 方法基于 Boltzmann 分布得到了拟合参数的构象集, 最后利 用遗传算法得到了描述粗粒化粒子间相互作用的 GB 参数. 结合利用量化计算得到的 EMP 参数, 我们 就获得到了精确描述两种有机分子的 GB-EMP 参 数. 利用得到的粗粒化参数, 基于粗粒化模型对范 德华相互作用曲线和径向分布函数曲线进行的计
算表明粗粒化模型能以较高的效率从整体上重复 全原子的计算结果, 但是在某些细节方面, 粗粒化 模型不能很好地符合全原子的分子动力学模拟结 果, 这主要与目前工作描述相互作用的位点较少及 相互作用位点放置简单有关. 为此我们应在下一步 工作中增加相互作用位点, 并应适当地调整GB 位 点和 EMP 位点的具体位置.

\section{References}

(1) Dror, R. O.; Jensen, M. Ø.; Borhani, D. W.; Shaw, D. E. J. Gen. Physiol. 2010, 135, 555.

(2) Karplus, M.; McCammon, J. A. Nature Struct. Biol. 2002, 9, 646.

(3) Shaw, D. E.; Maragakis, P.; Lindorff-Larsen, K.; Piana, S.; Dror, R. O.; Eastwood, M. P.; Bank, J. A.; Jumper, J. M.; Salmon, J. K.; Shan, Y. B.; Wriggers, W. Science 2010, 330, 341.

(4) van Gunsteren, W. F.; Bakowies, D.; Baron, R.; Chandrasekhar, I.; Christen, M.; Daura, X.; Gee, P.; Geerke, D. P.; Glättli, A.; Hünenberger, P. H.; Kastenholz, M. A.; Oostenbrink, C.; Schenk, M.; Trzesniak, D.; van der Vegt, N. F. A.; Yu, H. B. B. Angew. Chem. Int. Edit. 2006, 45, 4064.

(5) Klepeis, J. L.; Lindorff-Larsen, K.; Dror, R. O.; Shaw, D. E. Curr. Opin. Struct. Biol. 2009, 19, 120.

(6) Freddolino, P. L.; Harrison, C. B.; Liu, Y. X.; Schulten, K. Nature Phys. 2010, 6, 751.

(7) Xu, X. J.; Hou, T. J.; Qiao, X. B.; Zhang, W. Computer Aided Drug Design; Chemical Industry Press: Beijing, 2004; pp 169-172. [徐䈗杰, 侯廷军, 乔学斌, 章 威. 计算机辅助药 物分子设计. 北京: 化学工业出版社, 2004: 169-172.]

(8) Leach, A. P. Molecular Modeling, Principles and Application; Person Education Limited: England, 2001; pp 165-245.

(9) Schlick, T. Molecular Modeling and Simulation: An Interdisciplinary Guide, 2nd ed.; Springer: New York, 2010; pp 265-343.

(10) Voth, G. A. Coarse-Graining of Condensed Phase and Biomolecular Systems; CRC Press: England, 2009.

(11) Chu, J. W.; Izvekov, S.; Voth, G. A. Mol. Sim. 2006, 32, 211. 
(12) Marrink, S. J.; de Vries, A. H.; Mark, A. E. J. Phys. Chem. B 2004, 108, 750 .

(13) Tozzini, V. Curr. Opin. Struc. Biol. 2005, 15, 114.

(14) Golubkov, P. A.; Ren, P. Y. J. Chem. Phys. 2006, 125, 064103.

(15) Berne, B. J.; Pechukas, P. J. Chem. Phys. 1972, 56, 4213.

(16) Gay, J. G.; Berne, B. J. J. Chem. Phys. 1981, 74, 3316.

(17) Cleaver, D. J.; Care, C. M.; Allen, M. P.; Neal, M. P. Phys. Rev. E 1996, 54, 559.

(18) Wilson, M. R. J. Chem. Phys. 1997, 107, 8654.

(19) Care, C. M.; Cleaver, D. J. Rep. Prog. Phys. 2005, 68, 2665.

(20) Paramonov, L.; Yaliraki, S. N. J. Chem. Phys. 2005, 123, 194111.

(21) Kabadi, V. N.; Steele, W. A. Ber. Bunsenges. Phys. Chem. 1985, 89,2 .

(22) Kabadi, V. N. Ber. Bunsenges. Phys. Chem. 1986, 90, 327.

(23) Jackson, J. D. Classical Electrodynamics, 3rd ed; John Wiley \& Sons Inc.: New York, 1999; pp 145-150.

(24) Applequist, J. J. Phys. A 1989, 22, 4303.
(25) Ponder, J. W. TINKER Molecular Modeling, Package 5.1; Washington University Medical School.

(26) Darden, T.; York, D.; Pedersen, L. G. J. Chem. Phys. 1993, 98, 10089 .

(27) Sagui, C.; Pedersen, L. G.; Darden, T. A. J. Chem. Phys. 2004, $120,73$.

(28) Ren, P. Y.; Ponder, J. W. J. Phys. Chem. B 2003, 107, 5933.

(29) Wang, J. M.; Wolf, R. M.; Caldwell, W. J.; Kollman, P. A.; Case, D. A. J. Comput. Chem. 2004, 25, 1157.

(30) Yang, L. J.; Tan, C. H.; Hsieh, M. J.; Wang, J. M.; Duan, Y.; Cieplak, P.; Caldwell, W. J.; Kollman, P. A.; Luo, R. J. Phys. Chem. B 2006, 110, 13166.

(31) Frisch, M. J.; Trucks, G. W.; Schlegel, H. B.; et al. Gaussian 03, Revision A.01; Gaussian Inc.: Pittsburgh, PA, 2003.

(32) Stone, A. J. J. Chem. Theory Comput. 2005, 1, 1128.

(33) Golubkov, P. A.; Wu, J. C.; Ren, P. Y. Phys. Chem. Chem. Phys. 2008, 10, 2050 . 